\title{
Optimizing Surrogate End Points for Preoperative Systemic Therapies in Non-Small Cell Lung Cancers
}

\author{
William N. William Jr., MD ${ }^{1}$ and Stephen G. Swisher, MD $^{2}$ \\ ${ }^{1}$ Department of Thoracic/Head and Neck Medical Oncology, The University of Texas MD Anderson Cancer Center, \\ Houston, TX; ${ }^{2}$ Department of Thoracic and Cardiovascular Surgery, The University of Texas MD Anderson Cancer \\ Center, Houston, TX
}

Adjuvant chemotherapy has become the standard of care treatment for good performance status patients after resection of locally advanced, non-small-cell lung cancers (NSCLCs), based on the results of large, randomized, phase 3 clinical trials. The absolute benefit in survival at 5 years conferred by adjuvant cisplatin-based chemotherapy is estimated at $5.4 \%$, as described in a metaanalysis including 5 trials with 4584 patients. ${ }^{1}$ Efforts to improve outcomes by intensifying systemic therapy with the use of molecular targeted agents, for example, are currently being proposed in cooperative group settings (Alchemist Trial), but challenges exist because adjuvant clinical trials often call for large sample sizes and require a long time to evaluate objective end points such as survival. By the time a conclusive adjuvant trial is completed with novel agents, the knowledge obtained may have become obsolete. Furthermore, only a small number of drugs can be tested in the adjuvant setting, from the vast number of potential therapeutics currently under development.

One approach to overcome the limitations of adjuvant NSCLC trials is the use of preoperative (neoadjuvant) rather than a postoperative (adjuvant) therapy strategy for drug development in resectable NSCLCs. ${ }^{2}$ The metaanalysis by the NSCLC Meta-Analysis Collaborative Group has recently demonstrated that preoperative chemotherapy confers an absolute improvement in overall survival at 5 years of $5 \%$ compared with surgery up front. ${ }^{3}$ Although the metaanalysis does not compare adjuvant versus

(C) Society of Surgical Oncology 2014

First Received: 30 April 2014;

Published Online: 16 May 2014

W. N. William Jr., MD

e-mail: wnwillia@mdanderson.org preoperative therapy, the benefit observed is in the same order of magnitude of what has been demonstrated with an adjuvant approach, thus suggesting that either preoperative or adjuvant cytotoxic treatments could be used to improve outcomes in patients with locally advanced NSCLCs.

As discussed elsewhere, one potential advantage of preoperative therapy is the opportunity to evaluate response as a surrogate marker of benefit from treatment and to use this parameter as an end point for systemic therapy trials for patients with resectable NSCLCs. ${ }^{2}$ We envision a strategy according to which novel systemic agents can be evaluated in small to medium-sized preoperative trials to screen for activity in selected or unselected patient populations. Such studies would use response to treatment as the primary end point, which can be determined within a few weeks of initiating therapy. This obviates the need to follow patients long term after surgical resection to asses recurrence or death, thus reducing the number of resources necessary to complete such trials. Agents that demonstrate promising activity in preoperative (neoadjuvant) trials can then be evaluated in larger, definitive adjuvant phase 3 studies using survival as an end point. The use of response to treatment to screen for efficacy of investigational drugs is dependent on the ability of response to predict long-term recurrence-free and overall survival, thus serving as a valid surrogate/intermediate marker. Hence, refining the method for response evaluation will become key for the successful implementation of preoperative-based drug development strategies.

We have recently demonstrated that a major pathologic response (as defined by $\leq 10 \%$ viable tumor cells in the surgical specimen after preoperative systemic therapy) is strongly correlated with long-term recurrence-free survival and overall survival. ${ }^{4}$ These findings have been reproduced by other groups, thus leading to the recommendation of use 
of major pathologic response as an end point to be consistently embedded in the design of preoperative trials. ${ }^{2}$

In this issue of the journal, van Gool et al. provide significant contributions to the field of response assessment after preoperative therapy by demonstrating that a reduction in ${ }^{18} \mathrm{~F}$-fluorodeoxyglucose (FDG) uptake on positron emission tomography scans after 3 weeks of erlotinib correlates more strongly with histopathologic response compared with tumor shrinkage evaluated by computed tomography. Moreover, tumors with an EGFR mutation had a lower mean FDG uptake at 3 weeks compared with tumors that were EGFR wild-type (in press). The implications of these findings are many: (1) these data provide further support to the use of a noninvasive method to predict histopathologic response to targeted agents; (2) the magnitude of reduction of FDG uptake may be a useful method to assist in discovering potential biomarkers of response by identifying the patients whose driver molecular alterations have been inhibited by a specific molecular targeted agent, thus warranting more extensive molecular profiling in search of the driver molecular events; (3) the findings justify further studies on the correlations of response by positron emission tomography-computed tomography with long-term treatment outcomes, serving as an alternative and/or adjunct method to histopathologic evaluation as a surrogate marker of efficacy.

In summary, the work by van Gool et al. adds further evidence of the feasibility of preoperative treatment strategies to rapidly assess novel targeted agents using imaging and histopathologic response criteria for assessment of efficacy of treatment. The comprehensive characterization of these end points has the potential to greatly streamline drug development in this setting using rational and more feasible approaches. These studies raise the enthusiasm of evaluating novel agents in patient populations for which few effective treatment options exist. With these strategies there is vast potential to rapidly develop novel therapeutics and produce meaningful improvements in long-term survival in resectable NSCLC.

ACKNOWLEDGMENT This work is supported in part by the Mayberry Memorial Foundation

\section{REFERENCES}

1. Pignon JP, Tribodet $\mathrm{H}$, Scagliotti GV, et al. Lung adjuvant cisplatin evaluation: a pooled analysis by the LACE Collaborative Group. J Clin Oncol. 2008;26:3552-9.

2. Hellmann MD, Chaft JE, William WN Jr, et al. Pathological response after neoadjuvant chemotherapy in resectable non-smallcell lung cancers: proposal for the use of major pathological response as a surrogate endpoint. Lancet Oncol. 2014;15:e42-50.

3. NSCLC Meta-Analysis Collaborative Group. Preoperative chemotherapy for non-small-cell lung cancer: a systematic review and meta-analysis of individual participant data. Lancet. 2014;383: 1561-71.

4. Pataer A, Kalhor N, Correa AM, et al. Histopathologic response criteria predict survival of patients with resected lung cancer after neoadjuvant chemotherapy. J Thorac Oncol. 2012;7:825-32. 